\title{
Mobile Node Discovery Auxiliary Communication of 6LoWPAN
}

\author{
Fan Tongrang ${ }^{1}, \mathrm{He} \mathrm{Bingchao}^{2}$, Zhao Wenbin ${ }^{3 *}$, Huang Xin ${ }^{4}$ and $\mathrm{Yu} \mathrm{Tao}^{5}$ \\ ${ }^{1,2,3,4}$ School of Information Science and Technology, Shijiazhuang Tiedao University, \\ Shijiazhuang, Hebei 050043, China \\ ${ }^{5}$ Institute of Network Science and Cyberspace, Tsinghua University, Beijing, 100084, \\ China \\ lfantr@stdu.edu.cn, 3*253897826@qq.com
}

\begin{abstract}
Based on mobility support of IPv6 and node discovery mechanism, this paper proposes a method using node discovery mechanism for mobile node auxiliary connection, in which we detect the real-time received signal strength indicator, by computing and analyzing the change of average value to predict variation trend. In addition, we drew up a new generation of Internet protocol implementation scheme in 6LoWPAN, which is the access of the combination of the mobile node and IoT gateway, etc. In the local IoT, things connect with IPv6, which satisfy the demand of mobility, the whole IoT architecture model of IPv6 connection will be implemented. The analysis and evaluation of network performance was completed in Cooja simulator based on Contiki system after the hardware test. Results show that this method is suitable for the stub network of Internet of things in the common dynamic scenes and more efficient and less packet loss probability when compare to the original 6LoWPAN.
\end{abstract}

Keywords: Internet of Things, Mobility, Neighbor Discovery, 6LoWPAN, Contiki, Cooja

\section{Introduction}

In the year 1995, Bill Gates first proposed the concept of Internet of things in his book "The Road Ahead", after that, although the earliest Internet of Things (IoT) Concept has been proposed, but under the limitation of various wireless hardware and software, as well as the development of sensor technology, Internet of Things did not receive enough attention. However, The Internet of things, which developed up to now, has been integrated with IPv6, artificial intelligence, sensors, cloud computing, smart devices and many other technologies and applied to fields that are closely related to human activities, furthered human understanding and changing of nature process. Things can be seen as the integration of information and physical world [1]. Connections between human to human, human to things and things to things will become pervasive networks or ubiquitous networks by MultiNetworks and Multi-Technology's merge together [2]. With the enrichment of physical information world and Internet technology, topology trend to become high dynamics, which exposes mobility problems. Making efficient mobile node hand off and data transmission problems urgent to be solved.

Based on the Support for mobility of next generation Internet Protocol IPv6 (Internet Protocol Version 6) by nature [3] and previous studies of 6LoWPAN (IPv6 over Low-power Wireless Personal Area Networks) Network [4], as well as the Research on interoperability issues between Internet of things and IPv6 [5], we present a mobile nodes auxiliary communication method utilizing 6LoWPAN node discovery mechanisms and signal strength detection. This method further improved the efficiency of mobile hand off in Internet of Things and data transmission performance. 


\section{Related Work}

In the RFC3775 document has pointed out something about the description of IPv6 for mobility support. In IPv6, it is required that the protocol has good support for mobility, so in the article [6] put forward MIPv6 protocol. MIPv6 protocol enables nodes to be switched in the IPv6 network mobile, so that the nodes can still maintain the reachable state.

The literature [7] pointed out that in the research of low power network access to the Internet, in the 802.15.4 protocol, the multicast function is not fully realized, but it has the characteristics of overlapping broadcast domains.

The literature [8] has evaluated the mobile IPv6 based on Contiki [9], then obtained the mobile IPv6 can be a method of solving the problem of network mobility in 6LoWPAN.

Although the mobile IP management scheme is simple and feasible, it still has some defects. The mobile handoff scheme based on the proxy is improved [10], and the speed of the topology reconstruction is improved by the home access point in the process of fast handover of the mobile nodes. The literature [11] proposed to improve the discovery mechanism in 6LoWPAN, designed an extended 6LoWPAN neighbor discovery protocol, and some improvement ideas are given out from the original protocol.

In order to effectively use IPv6 technology in wireless sensor networks, the 6LoWPAN working group has overcome some difficulties, and has made great progress in the field. Although the IPv6 itself provides support for mobility, mobility in wireless sensor networks is still an open problem.

In addition, the paper [12] also evaluated the mobile IPv6 on 6LoWPAN and realized mobile IPv6 experiment on Contiki on the real experimental platform, and achieved a new mobile detection mechanism based on passive listening.

In 6LoWPAN, the neighbor discovery process is embodied in the RPL protocol, the topology building process of RPL is also based on the discovery of neighboring nodes [13]. By controlling the networking messages, it can improve the performance of the network. From this point of view, so as to make optimization of node mobile application.

In order to analyze the general behavior of RPL under different conditions. Xie H [14] conducted a wide range of simulation. Sensor nodes are dispersed within the sensing range, from the characteristics of these simulations, can be learned, Trickle timer, routing overhead, average end to end delay and other information.

The storage overhead of RPL routing in LLN networks is proposed by Yang Hong et al. [15]. An improved B-RPL routing protocol is proposed, which simplifies the routing table for the destination node, which greatly reduces the storage cost. In addition, the improved protocol also includes a new adaptive mechanism for dynamic topology, which is only a very small communication overhead.

Resolution Algorithm Batch (BRA) is a group of nodes (batch processing), which is a group of nodes (batch processing). Zanella proposed a new solution algorithm, adaptive batch feedback time algorithm [16].

Some researchers have pointed out that the access control of the Internet of things, the network access authentication protocol (PANA) has been standardized by the Internet Engineering Task Force, which can be carried out by the extensible authentication protocol (EAP). Literature [17] is the first time in the relevant field of low energy equipment EAP/PANA protocol feasibility study. Contiki system equipped with the device to provide an interactive EAP/PANA protocol, known as PANATIKI.

About the problem of 6LoWPAN node mobility, the existing research focuses on the use of network based mobile protocol, and the routing mechanism, mobility, security, etc., to add to each node is low feasibility, however, adding the routing mechanism, mobility, security, etc. to each node is low feasibility. Therefore, these functions should be delegated to the network, so that the sensor nodes focus on sensing environment, transmitting data, controlling and other functions, so as to reduce the energy consumption and delay caused by mobile 
switching [18]. In this paper, the mobile switching function is mainly realized on the access node or the Internet of things gateway, and the load of the terminal is limited.

\section{6LoWPAN ND Auxiliary}

6LoWPAN has Short-distance transmission and low-power characteristics, meaning it is suitable for mobile terminal networks and the field of embedded IPv6 [19]. It not only can make tremendous devices networking each other but also can be controlled by IPv6 protocol access to Internet. 6LoWPAN nodes use IPv6 protocol access to the internet, make a lot of IoT and Internet nodes have equal status [20]. Due to the characteristic of the short distance communication, 6LoWPAN has relatively higher density when compare with other network topology, which means the overlapping communication area is larger. Then transport messages through multi-hop and implement message delivery in 6LoWPAN network.

\subsection{Auxiliary Communication Process}

Neighbor discovery mechanism is the basic component of IPv6 protocol, its main functions include routing and prefix discovery, address resolution, reachability testing, redirection, etc. [21]. 6LoWPAN link layer does not support multicast, relying on the algorithm by means of unreliable broadcast multicast will consume a lot of energy [22]. Watteyne described the development of the network, the traditional neighbor discovery has been extended, especially in the emergence of a highly efficient low power wireless protocol ND Wireless (WiND), which extends the node registration mechanism [23].

In the network layer of the scheme, the connection between the nodes of 6LoWPAN and the data transfer process are optimized, which is the process of establishing and maintaining the topology. Starting from RPL, the connection and maintenance of RPL topology is divided into two processes. One is the node discovery phase of the RPL topology, and the two is the transmission phase of the data between nodes.

Mobile switching and data transfer in a mobile communication process as shown in Figure 1. Finally, through the Cooja test network self-healing ability, data transmission coherence and node energy consumption.

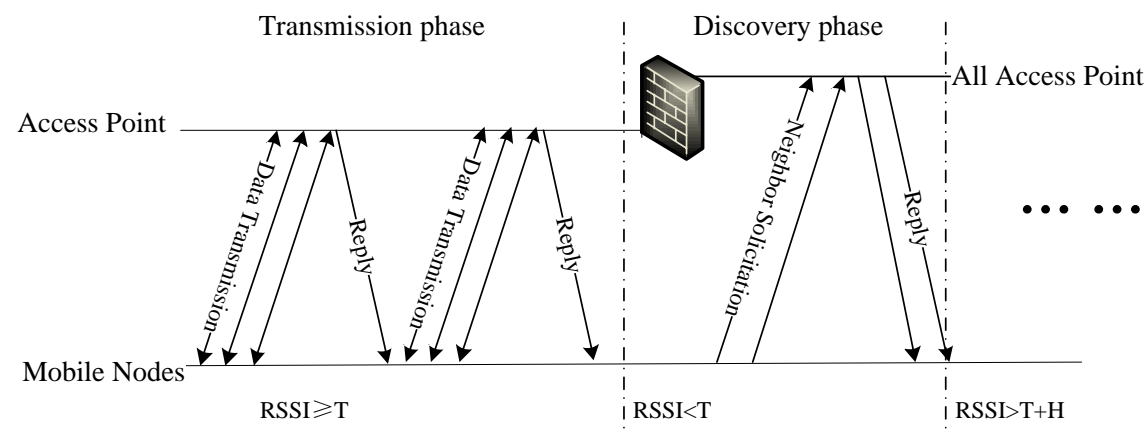

Figure 1. Communication Process

In RPL protocol, the root node is usually used as a border router or access node in the network, all nodes automatically create a neighbor table and maintaining the routing table. Opening the RPL protocol only needs to put a node as the root node, then initiates the establishment of destination-oriented directed acyclic graph topology. To build one or more DODAG, in the formation process of this topology, there are three kinds of messages: DIO (DODAG Information Object) DODAG information object, DAO (Destination Advertisement Object) DODAG object of destination announcement and DIS (DODAG Information Solicitation), topological information request. First, the root node sends DIO messages, put the related information in the network topology inform neighbor nodes, the 
node that receives the DIO message according to the information in the message to determine whether to join the network, if the node joins the topology, send DAO message which contains its own prefix information to the root node, and calculate its own rank value, it is equivalent to the depth of the tree structure, then update the DAO message, then continue to send multicast DIO messages to other nodes in the network. In addition, the nodes in the network can also initiate active applications by DIS messages, gather DIO messages and join the network topology. So need send DAO messages to the root node when a node is added to the topology or the topology changes. Each node is looking for its parent node in DODAG in the neighbor discovery process, then put it as the default upper node, at the same time, it also establishes and maintains a routing table that stores the list of its child nodes.

The algorithms of node transmission and discovery phase are as follows:

Transmission phase:

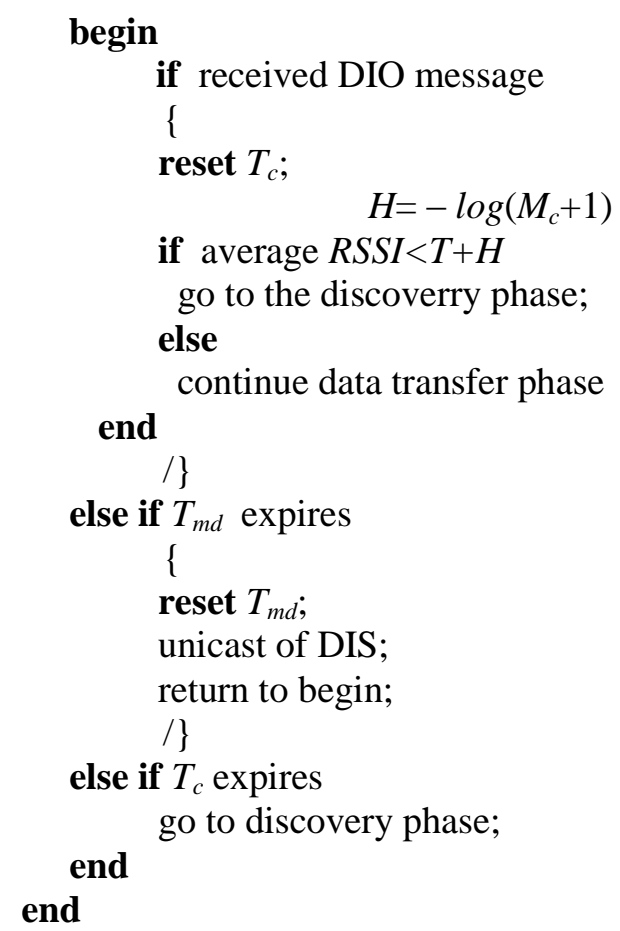

1) Discovery phase:

$$
\text { begin }
$$

if recieved DIS msg

\{

store RSSI values;

store counter value $C$ of the latest DIS message;

reset $T_{r}$ with $(N-C) * T_{d i s}$;

if $T_{r}$ expires

\{

calculate average RSSI;

send unicast DIO msg with average RSSI;

/\}

else

countinue discovery phase;

end

Else

/\}

if $T_{m c}$ expires 


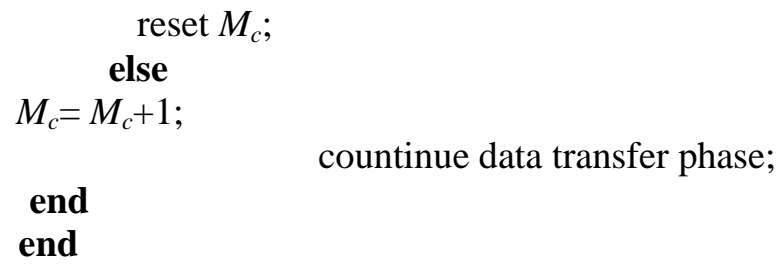

Among the algorithm: RSSI is the received signal strength received by the node; Tc is connected with the timer, in the timing phase, it continuous listening channel; Mc is the value of set mobile meter, through its change to maintain the stability of the network, it can effectively prevent the occurrence of severe mobile link failure from network violent mobile; Tmd is node mobile probe timer, depending on the frequency of the mobile node to send data to change, the higher the rate of data generated, the shorter the time, and request unicast DIO message for access points at the end of the time, and determination average signal intensity everytime; Tr is reply timer; Tdis is the time of DIS message interval; $\mathrm{N}$ is the internal number of custom data packet, it is also the number of packets required to calculate the average signal strength, the value is determined according to the data generation rate, and the same time, it influences the stability of network.

\subsection{Test and Experiment}

In order to obtain more accurate statistics in detail, we carried on the real environment message delivery of network communication test and experiment, then we simulate the solution in large scale through Cooja simulator.

Using several SM2530 [24] nodes, i.e. Smart Mote 2530, which employ TI (Texas Instruments) CC2530F256 wireless single chip SOC. It integrate 8051 kernal SCM, IEEE802.15.4 standard RF and 256KB ROM and 8KB SRAM, as well 128byte transmitreceive FIFO. Base on Contiki system, we program and transplant protocol stacks making the nodes different functional, such as IoT gateway, border router, fixed host and mobile node. Testing and make sure the connectivity between 6LoWPAN terminal node and the sink node and Internet.

Setup a communication area with overlapping zone using 4 fixed nodes, in addition to this, we need a mobile node, which moves through the overlapping field. The related information and packets could be obtained by serial port output or sniffer. Then we analyze the change of topology and information transmission situation.

Designing initial network topology structure and Adopting reasonable network scale, to make it fully embodies the mobility of network. Then we use Cooja mobility plugin and import related configuration file. Simulating the solution in reasonable scale through Cooja simulator so that we can analyze the mobility performance and record logs of the movement circumstance of the mobile nodes.

Analyze the mobility solution performance and then compare with original 6LoWPAN. 


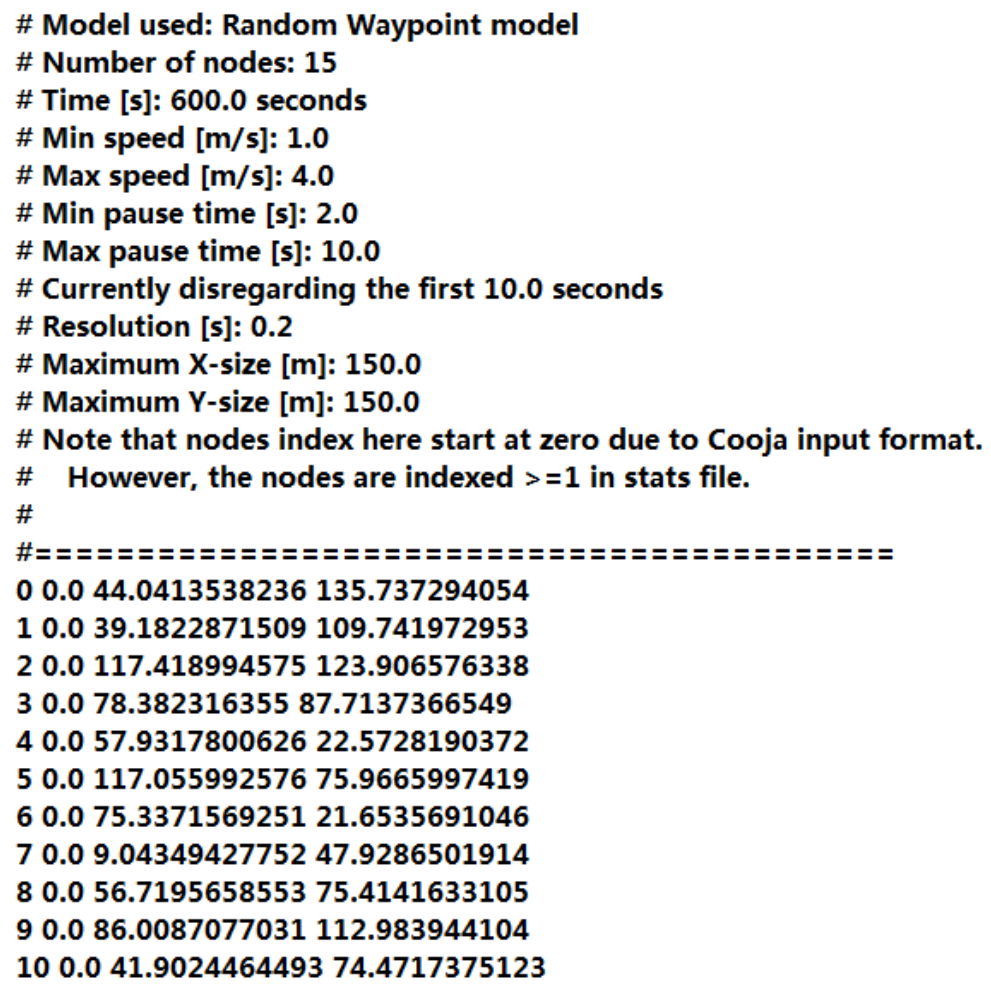

\section{Figure 2. Nodes Motion Trail Configuration}

After the real environment experiment test, we conduct simulation experiment in Cooja, in order to obtain more accurate and comprehensive information. Nodes mobility support in Instant Contiki has been implemented [25]. The traverse route can be designed by compiling position.dat configuration file. Such as figure 2, values in the first column represent for node serial number, and in second column, they are certain time in movement. The last two columns indicate nodes coordinates $\mathrm{x}$ and $\mathrm{y}$ in a field.

During the test, first, configure and test nodes of 6LoWPAN, design reasonable topology configuration, and initial IPv6 address. RPL protocol using automatic networking, then 6LoWPAN normal communication must be confirmed.

Secondly, through the application of Contiki system in the virtual serial port to upload data to the local computer, we can observe the working state between nodes, figure 3 is an UDP communication sample between two nodes, and we can view the output RSSI information of the packet node. 


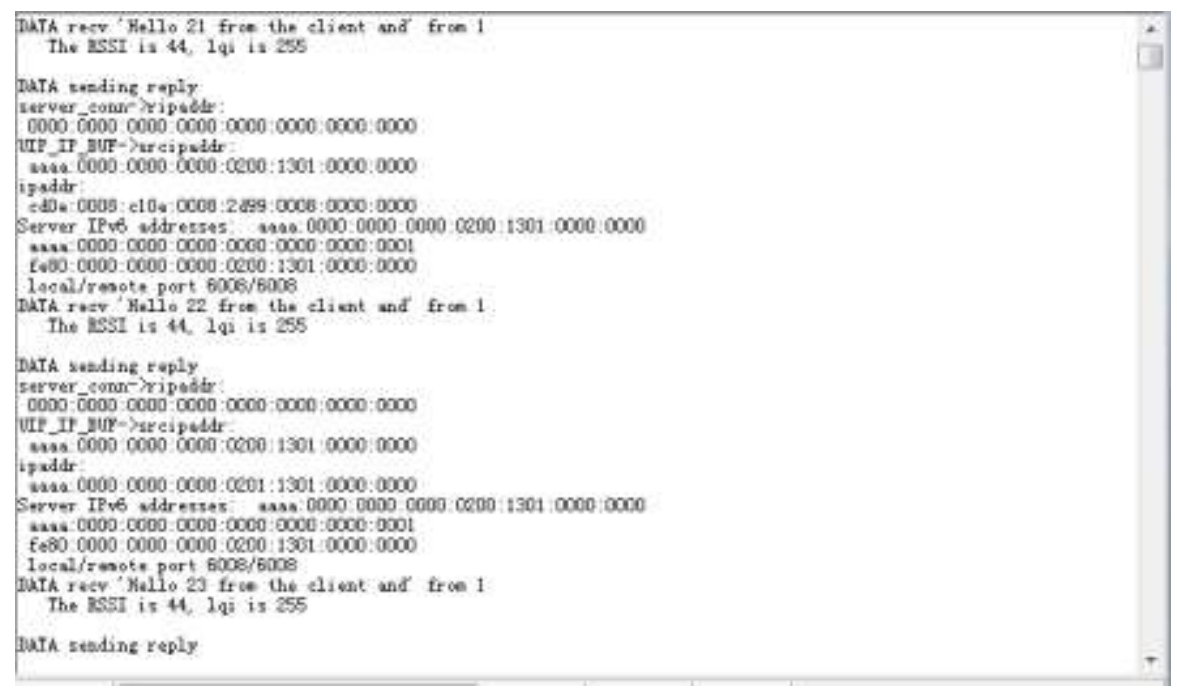

Figure 3. Communication State

Finally, import the motion state into the nodes, and then restart the simulation, nodes will be moving in the setup state, and nodes according to the order of the corresponding configuration file serial number when joining. When the time of periodic motion set is expired, all nodes will start from the initial state, so the cycle continues.

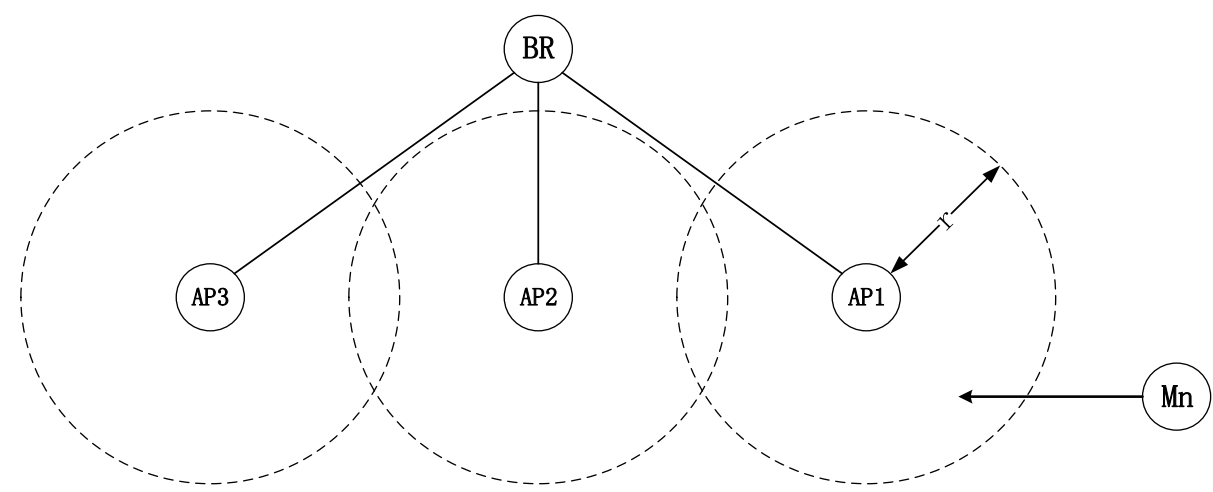

Figure 4. Experimental Scene Configuration

In the test of the performance of the network organization, after successful network organization test, then test the capability of communication in $1 \mathrm{~m}$ within the communication range of static state nodes, which act as the reference. Set a sniffer capture node in the network that connected by pipes with Wireshark [26]. Capture and analyze network packets. Considering the 802.15.4 standard communication range of theory is about $100 \mathrm{~m}$, so we adjust the transmit power, change default emission power 4.5dBm (TX Tx_Power:0xF5) to 6dbm (TX Tx_Power:0x85), so that it can more clearly reflect the network performance parameters. The communication coverage radius $\mathrm{R}$ that we set is $50 \mathrm{~m}$, average RSSI threshold is set to 10 . The experimental scene configuration diagram is as follows:

Then, the network parameters of the $1 \mathrm{~m}$ to $40 \mathrm{~m}$ indoor environment experiment was conducted, and the network performance of $1 \mathrm{~m} / \mathrm{s}, 5 \mathrm{~m} / \mathrm{s}, 10 \mathrm{~m} / \mathrm{s}$ mobile and static states were tested. After that, we analyzed the comparison of the original protocol and our solution under the application of UDP communication, the results are given in the next section.

\section{Experimental Result Analysis}

The data statistic of the 6LoWPAN network has been changed in the static and dynamic situations, including percentage of network control information, access delay, data 
transmission loss rate, energy consumption, etc. Figure 5 and Figure 6 is statistics on the percentage of control messages in the network integrated transceiver packed respectively in the near static state and the $10 \mathrm{~m}$ range of the average $5 \mathrm{~m} / \mathrm{s}$ uniform mobile state. In the experiment, there is a certain chance, such as the influence of the external objects or signal interference, but a large number of statistical results can reflect the changes of the network performance. Due to the use of a similar mechanism for the pre-sentence, in general static situation, the test control message in the network is increased compared with the existing situation, and it will increase the energy consumption. However, in the dynamic situation, the situation is reversed, limited by the original protocol performance, the network delay and packet loss rate of the original protocol are beginning to rise, so as to maintain the stability of the network.

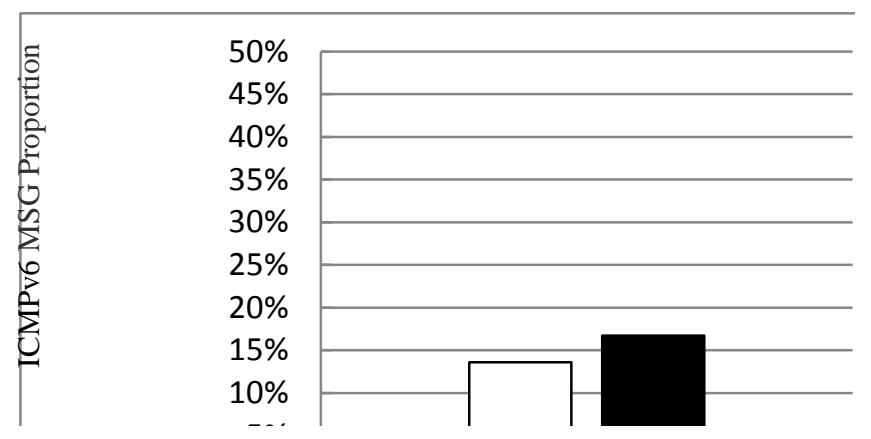

Figure 5. Percentage of Control Messages (static)

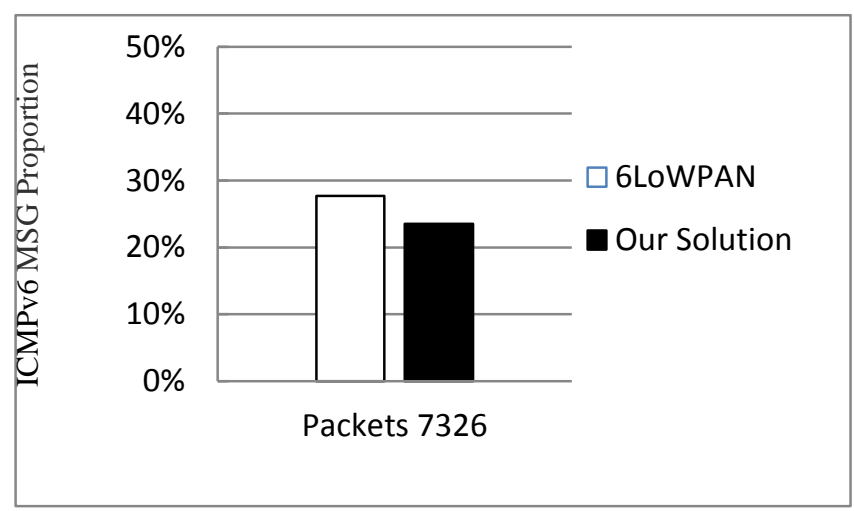

Figure 6. Percentage of Control Messages (static)

As shown in Figure 7 and figure 8, by using the method of neighbor discovery mechanism auxiliary shift, the probability of network switching error is reduced, the stability of network and the transmission efficiency of the network is increased, and in the case of mobile scene of large energy consumption, the energy consumption and transmission efficiency can be better controlled obviously. 


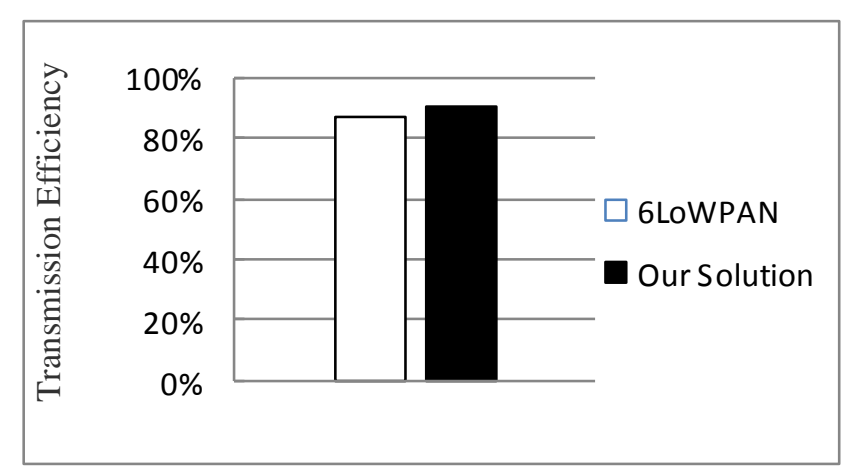

Figure 7. Transmission Efficiency of Data Packet

Through statistical analysis of network access delay under various conditions, it can be concluded that the performance of the two methods is less different in the static or low speed condition, call the original protocol more stable and fast. However, in the high-speed mobile state, the advantages is obvious by using RPL to support the pre handoff method, compared with the original method, there are about $20 \%$ of the speed improving, and the access efficiency is stable with the increase of speed.

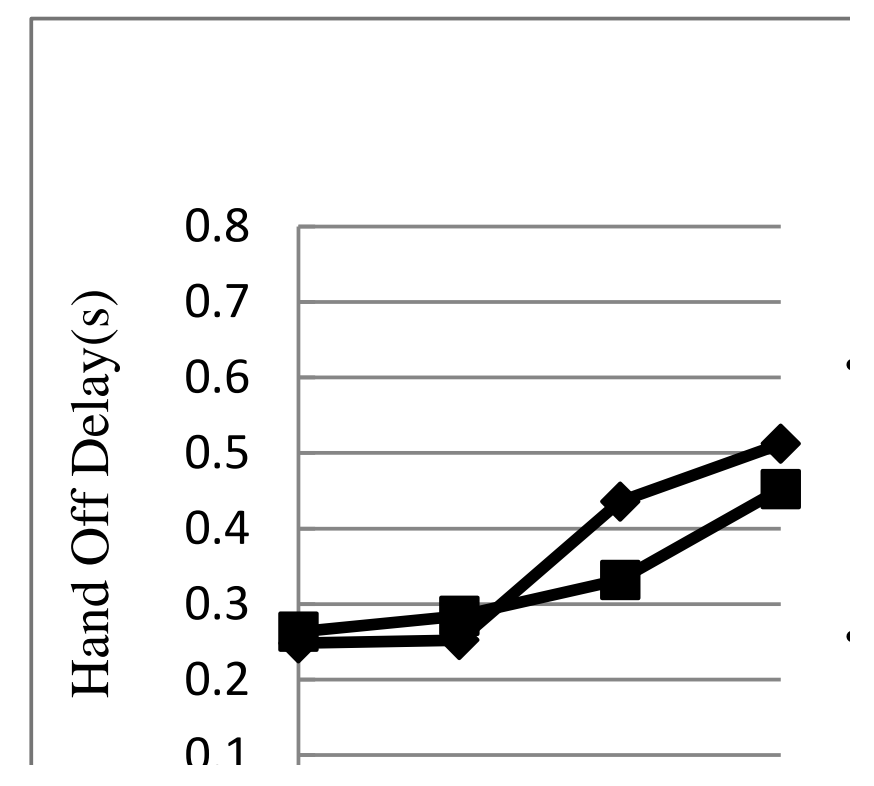

Figure 8. Delay of Handoff Access

\section{Conclusion}

With the next generation network and the technology of low power network promoting and popularizing continuously, 6LoWPAN has gradually replaced the ZigBee, it becomes a solution of Thing nodes interconnection and interworking with the way of the IPv6. Due to the characteristic of the short distance communication, 6LoWPAN usually been used as a network solution of Things end nodes, and the problem of mobile management is the key to solve the performance of the end of the internet of Things. Through the exploration and experiment to the realization method of 6LoWPAN nodes efficient mobile handoff communication, this paper gives a method for nodes movement and access management in the local IoT. We have confirmed its feasibility and efficiency from the real experimental platform and a certain scale of the simulation experiment. Besides, this paper proposes the idea of using the way of compatible IPv6 to integration with networking and internet. It helps to promote the application of the internet of things. 6LoWPAN technology has good 
development prospects and research value, and the improvement in terms of mobile performance can promote its wide application in natural monitoring, industrial control, smart home, experiment teaching and other fields.

\section{Acknowledgments}

This study is funded by the National Natural Science Foundation of China (\#61373160).

\section{References}

[1] X. Zhu, L. Qi and J. Sun, "Internet of Things and Ubiquitous Communication Technology", Posts \& Telecom Press, (2010).

[2] S. Qibo, L. Jie, L. Shan, F. Chunxiao and S. Juanjuan, "Internet of Things: Summarize on Concepts,Architecture and Key Technology Problem", Journal Of Beijing University of Posts and Telecommunications, vol. 33, no. 3, (2010), pp. 1-9.

[3] C. Lin and L. Lei, "Research on Next Generation Internet Architecture", Chinese Journal of Computers, vol. 30, no. 5, (2007), pp. 693-711.

[4] W. Hequan. "IOT application and challenges: a review", Chongqing University of Posts and Telecommunications Journal: Natural Science Edition, vol. 22, (2010), pp. 526-531.

[5] S. Hossen, A. F. M. S. Kabir, R. H. Khan and A. Azfar, "Interconnection between 802.15.4 Devices and IPv6: Implications and Existing Approaches", International Journal of Computer Science Issues, vol. 7, no. 1, (2010), pp. 19-31.

[6] D. Johnson, C. Perkins and J. Arkko, "RFC 3775: Mobility support in IPv6", IETF, (2004).

[7] A. Terzis, S. D. Haggerty, D. E. Culler, J. W. Hui and P. Levis, "Connecting low-power and lossy networks to the internet", Communications Magazine, IEEE, vol. 49, no. 4, (2011), pp. 96-101.

[8] J. Montavont, D. Roth and T. Noel, "Mobile IPv6 in Internet of Things: Analysis, experimentations and optimizations", Ad Hoc Networks, vol. 14, no. 2, (2014), pp. 15-25.

[9] A. Dunkels, B. Gronvall and T. Voigt, "Contiki-a lightweight and flexible operating system for tiny networked sensors", Local Computer Networks, 2004, 29th Annual IEEE International Conference on IEEE, (2004), pp. 455-462.

[10] Y. F. Zhang and Q. Z. Zhang, "Based fast handover for proxy mobile IPv6 route optimization scheme", Computer applications, vol. 32, no 2, (2012), pp. 335-339.

[11] M. K. Shin and H. J. Kim, "Mobility support in IP-based sensor networks", ICT Convergence (ICTC), 2011 International Conference on. IEEE, (2011), pp. 567-568.

[12] J. Lee, H. K. Kang, D. S. Lim, C. S. Hong and S. Lee, "An ID/Locator Separation-Based Mobility Management Architecture for WSNs", Mobile Computing, IEEE Transactions on, vol. 13, no 10, (2014), pp. 2240-2254.

[13] P. Thubert, T. Winter, A. Brandt, J. Hui, R. Kelsey and P. Levis, "RPL: IPv6 Routing Protocol for LowPower and Lossy Networks", Internet Requests for Comment, (2012), rfc 6550.

[14] G. Zhang, D. Su, P. Wang and F. Zeng, "Performance evaluation of RPL routing protocol in 6lowpan", Software Engineering and Service Science (ICSESS), (2014) 5th IEEE International Conference on. IEEE, (2014), pp. 625 - 628.

[15] Y. Hong, S. Limin and Z. Hongsong. "B-RPL: low storage overhead of RPL routing protocol", Computer Science, vol. 42, no. 1, (2015), pp. 96-100.

[16] Zanella and Andrea, "Adaptive Batch Resolution Algorithm with Deferred Feedback for Wireless Systems", Wireless Communications, IEEE Transactions on, no. 10, (2012), pp. 3528-3539.

[17] M. S. Pedro, M. L. Rafa and A. F. G. Skarmeta, "PANATIKI: a network access control implementation based on PANA for IoT devices", Sensors, vol. 13, no. 11, (2013), pp. 14888-14917.

[18] N. Kushalnagar, G. Montenegro and C. Schumacher, "IPv6 over Low-Power Wireless Personal Area Networks (6LoWPANs): Overview, Assumptions, Problem Statement, and Goals", Heise Zeitschriften Verlag, (2007), pp. 1-12.

[19] M. Mao M, "Research and implementation of 6LoWPAN adaptation layer and ND protocol", East China Normal University, (2007), pp. 8-76.

[20] B. He, S, Di and T. Fan, "Mobile Node Discovery Auxiliary Communication of 6LoWPAN", Journal Of HeBei Academy of Sciences, vol. 31, (2014), pp. 74-78.

[21] Zhao, Wenbin, Zhao, Zhengxu, "Research on engineering software data formats conversion network", Journal of Software, vol. 7, no. 11, (2012), pp. 2606-2613.

[22] U. Nagaraj and D. G. Deotale, "IPv6 Multicast in VANET", International Journal of Computer Science \& Information Security, vol. 10, iss. 4, (2012), p. 135.

[23] T. Watteyne and P. Thubert, "Efficient 6LoWPAN Neighbor Discovery applied to Multilink IoT subnets", Communications (ICC), 2015 IEEE International Conference on. IEEE, (2015), pp. 642-647.

[24] Texas Instruments Incorporated, CC253x System-on-Chip Solution for 2.4-GHz IEEE 802.15 .4 and ZigBee®Applications[EB/OL].http://www.godsp.com/forms/techdoc/doc_feedback.htm?litnum=SWRU191 C, Literature Number: SWRU191C April 2009-Revised January (2012). 
[25] F. Klink, "Mobility Cooja Plugin". https://github.com/contiki-os/contiki/wiki, (2014).

[26] A. Orebaugh, G. Ramirez and J. Beale, "Wireshark \& Ethereal network protocol analyzer toolkit", Syngress, (2006).

\section{Authors}

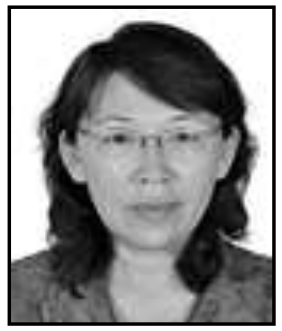

Fan Tong-rang, she was born in 1965, Professor. Ph.D. School of Information Science and Technology, Shijiazhuang Tiedao University. Her main research interest includes network technology and Information processing. Email address: fantr@stdu.edu.cn; fantr2009@126.com.

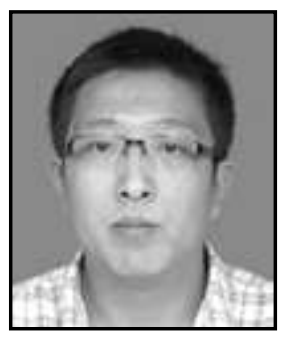

Zhao Wen-bin, he was born in 1985, Ph.D. School of Information Science and Technology, Shijiazhuang Tiedao University. His major field of study is network technology and information processing. Email address: 253897826@qq.com. 
International Journal of Future Generation Communication and Networking Vol. 10, No. 1 (2017) 